\title{
A note on commuting graphs for general linear groups
}

\author{
Maryam Nasiri (D), Sayyed Heidar Jafari* (1D \\ Department of Mathematics, Shahrood University of Technology, Shahrood, P.O. Box: 316-3619995161, \\ Iran
}

\begin{abstract}
Let $G$ be a group and $X$ a subset of $G$. Then $\mathcal{C}(G, X)$ is a graph with vertex set $X$ in which two distinct elements $x, y \in X$ are joined by an edge if $x y=y x$. In this paper, we study the clique number, the domination number, the diameter, the planarity, the perfection and regularity of $\mathrm{C}(G, X)$ where $G=G L(n, q)$ and $X$ is the set of transvections.
\end{abstract}

Mathematics Subject Classification (2010). 20D99, 05C69, 05C25, 20G40

Keywords. commuting graph, transvections, clique, domination, perfection, planarity

\section{Introduction and preliminaries}

We consider simple graphs which are undirected, with no loops or multiple edges. For any graph $\Gamma$, we denote the sets of the vertices and edges of $\Gamma$ by $V(\Gamma)$ and $E(\Gamma)$, respectively. A graph $\Gamma$ is regular if all the vertices of $\Gamma$ have the same degree. A subset $X$ of $V(\Gamma)$ is called a clique if the induced subgraph on $X$ is a complete graph. The maximum size of a clique in a graph $\Gamma$ is called the clique number of $\Gamma$ and is denoted by $\omega(\Gamma)$. A subset $X$ of $V(\Gamma)$ is called an independent set if the induced subgraph on $X$ has no edges. The maximum size of an independent set in a graph $\Gamma$ is called the independence number of $\Gamma$ and is denoted by $\alpha(\Gamma)$. A $k$-vertex colouring of a graph $\Gamma$ is an assignment of $k$ colours to the vertices of $\Gamma$ such that no two adjacent vertices have the same colour. The vertex chromatic number $\chi(\Gamma)$ of a graph $\Gamma$, is the minimum $k$ for which $\Gamma$ has a $k$-vertex colouring. For a graph $\Gamma$ and a subset $S$ of the vertex set $V(\Gamma)$, denote by $N_{\Gamma}[S]$ the set of vertices in $\Gamma$ which are in $S$ or adjacent to a vertex in $S$. If $N_{\Gamma}[S]=V(\Gamma)$, then $S$ is said to be a dominating set of vertices in $\Gamma$. The domination number of a graph $\Gamma$, denoted by $\gamma(\Gamma)$, is the minimum size of a dominating set of vertices in $\Gamma$. The length of the shortest cycle in a graph $\Gamma$ is called the girth of $\Gamma$ and denoted by $\operatorname{girth}(\Gamma)$. If $v$ and $w$ are vertices in $\Gamma$, then $d(v, w)$ denotes the length of the shortest path between $v$ and $w$. The largest distance between all pairs of the vertices of $\Gamma$ is called the diameter of $\Gamma$, and is denoted by $\operatorname{diam}(\Gamma)$. A graph $\Gamma$ is connected if there is a path between each pair of vertices of $\Gamma$. A planar graph is a graph that can be embedded in the plane so that no two edges intersect geometrically except at a vertex to which both are incident. A graph $\Gamma$ is called perfect if for every induced subgraph $H$ of $\Gamma, \omega(H)=\chi(H)$, and $\Gamma$ is Berge if

\footnotetext{
*Corresponding Author.

Email addresses: nasirimaryam.63@gmail.com (M. Nasiri), shjafari55@gmail.com (S.H. Jafari)

Received: 18.09.2017; Accepted: 23.05.2018
} 
no induced subgraph of $\Gamma$ is an odd cycle of length at least five or the complement of one. The following theorems and definitions will be used repeatedly.

Theorem 1.1. [2, Theorem 1.2] A graph is perfect if and only if it is Berge.

Definition 1.2. [4, Definition and Theorem 8.5] If $V$ is an n-dimensional vector space over a field $F$, then the general linear group $G L(V)$ is the group of all nonsingular linear transformations on $V$ with respect to the composition of mappings.

Choosing an ordered basis of $V$ gives an isomorphism $G L(V) \longrightarrow G L(n, F)$, where $G L(n, F)$ is the group of all invertible $n \times n$ matrices over $F$. If $F$ is finite, with $q$ elements, this group is denoted by $G L(n, q)$. Also

$$
|G L(n, q)|=\left(q^{n}-1\right)\left(q^{n}-q\right) \ldots\left(q^{n}-q^{n-1}\right) .
$$

The determinant function det $: G L(n, F) \longrightarrow F^{\star}$ is a homomorphism which maps the identity matrix to 1 , and it is multiplicative, as desired. The special linear group, $S L(n, F)$, is the kernel of this homomorphism. The center of $G L(V)$ is $Z(G L(V))=\left\{\lambda I: \lambda \in F^{\star}\right\}$ and the center of $S L(n, F)$ is $Z(S L(n, F))=\left\{\lambda I: \lambda \in F^{\star}, \lambda^{n}=1\right\}$. Define the projective general linear group and the projective special linear group on $V$ to be

$$
P G L(V)=\frac{G L(V)}{Z(G L(V))} \quad, \quad P S L(V)=\frac{S L(V)}{Z(S L(V))} .
$$

A hyperplane $W$ in $V$ is a subspace of dimension $n-1$. Let $W$ be a hyperplane of $V$. If $I \neq T \in G L(V)$ satisfies:

$$
T(w)=w \quad \forall w \in W, T(v)-v \in W \quad \forall v \in V
$$

then $T$ is called a transvection with respect to $W$ and $W$ is called the axis of the transvection $T$. For each transvection $T$, $\operatorname{det}(T)=1$. So $T \in S L(V)$. The inverse of a transvection is a transvection. The set of transvections generates $S L(V)$. Given a nonzero linear functional $f$ on $V$ and a nonzero vector $a \in \operatorname{ker} f$, define $T_{a, f}: V \longrightarrow V$ by $T_{a, f}: v \longmapsto v-f(v) a$. It is clear that $T_{a, f}$ is a transvection. Moreover, for every transvection $T$ there exist $f \neq 0$ and $a \neq 0$ with $T=T_{a, f}$.

Theorem 1.3. Let $V$ be an $n$-dimensional vector space over a field $F$. Then intersection of null space of $k$ independent linear functionals is an $(n-k)$-dimensional subspace of $V$.

Proposition 1.4. [4, Corollary 8.18 and Theorem 8.21] All transvections in $G L(n, F)$ are conjugate. If $n \geq 3$, then they are conjugate in $S L(n, F)$.

Lemma 1.5. [4, Lemma 8.19] Let $V$ be a vector space over $F . T_{a, f}=T_{b, g}$ if and only if there is a scalar $\alpha \in F^{\star}$ with $g=\alpha f$ and $a=\alpha b$.

For a hyperplane $W$ in a vector space $V$, we set $\tau(W)=\{$ all transvections fixing $W$ \}$\cup\left\{1_{V}\right\}$.

Lemma 1.6. [4, Lemma 8.22] Let $W$ be a hyperplane in an $n$-dimensional vector space $V$ over $F . \tau(W)$ is an abelian subgroup of $S L(V)$, and $\tau(W) \cong W$.

Definition 1.7. [1] A graph $\Gamma$ is vertex-transitive if the automorphism group of $\Gamma$ acts transitively on the vertex set of $\Gamma$.

Theorem 1.8. [1, Theorem 7.1] Let $\Gamma$ be a k-regular, connected, vertex-transitive graph of order $n$. Then

(1) If $n$ is even, then $\Gamma$ has a 1-factor.

(2) The product of the clique number and the independence number of $\Gamma$ is at most $n$. 


\section{Main result}

The purpose of this note is to study certain properties of the commuting graph $\mathcal{C}(G, X)=$ $\Gamma$ where $G=G L(n, q)$ and $X$ is the set of transvections in $G$. Throughout this paper $V$ is a vector space with $\operatorname{dim}(V)=n$ on a finite field $F$ with $|F|=q$.

Lemma 2.1. For a proper subspace $U$ of $V$, set $S_{U}=\left\{T_{a, f} \mid a \in U, U \subseteq \operatorname{ker} f\right\}$. Then $\left|S_{U}\right|=\frac{\left(q^{i}-1\right)\left(q^{n-i}-1\right)}{q-1}$, where $i=\operatorname{dim}(U)$.

Proof. We have $\{f: V \rightarrow F \mid U \subseteq \operatorname{ker} f\} \cong\left\{\bar{f}: \frac{V}{U} \rightarrow F\right\}$. So there are $q^{n-i}-1$ candidates for $f$, that is the number of nonzero linear functionals from $\frac{V}{U}$ into $F$, and $q^{i}-1$ candidates for $a$ (the zero vector is not a candidate). By Lemma 1.5, $\left|S_{W}\right|=\frac{\left(q^{i}-1\right)\left(q^{n-i}-1\right)}{q-1}$.

Lemma 2.2. $|V(\Gamma)|=\frac{\left(q^{n}-1\right)\left(q^{n-1}-1\right)}{q-1}$.

Proof. We consider a fixed hyperplane $W$. It is sufficient to calculate $\mid\left\{T_{a, f} \mid a \in W, W=\right.$ ker $f\} \mid$. Since the number of hyperplanes in $V$ is equal to $\frac{q^{n}-1}{q-1}$, we have $|V(\Gamma)|=$ $\frac{\left(q^{n}-1\right)\left(q^{n-1}-1\right)}{q-1}$ by Lemma 2.1 .

Lemma 2.3. [3, Lemma 1, part (iv)] Let $T_{a, f}$ and $T_{b, g}$ be two transvections on $V$ with fixed hyperplanes $W_{1}$ and $W_{2}$, respectively. Then $\left[T_{a, f}, T_{b, g}\right]=1$ if and only if $a \in \operatorname{ker} g$ and $b \in \operatorname{ker} f$.

Lemma 2.4. $\Gamma$ is k-regular with $k=\frac{(q-1)\left(q^{n-1}-1\right)+q\left(q^{n-2}-1\right)^{2}}{q-1}-1$.

Proof. By proposition $1.4, \Gamma$ is a $k$-regular graph. Let $T_{a, f}$ be a transvection. It is sufficient to calculate $|\{(b, g) \mid b \in \operatorname{ker} f, a \in \operatorname{ker} g\}|$. We have

$$
\begin{aligned}
|\{(b, g) \mid b \in\langle a\rangle, a \in \operatorname{ker} g\}| & =(|\langle a\rangle|-1)\left(\left|\left(\frac{V}{\langle a\rangle}\right)^{*}\right|-1\right) \\
& =(q-1)\left(q^{n-1}-1\right)
\end{aligned}
$$

and

$$
\begin{aligned}
|\{(b, g) \mid b \in \operatorname{ker} f, a \in \operatorname{ker} g,\langle a\rangle \neq\langle b\rangle\}| & =|\operatorname{ker} f-\langle a\rangle|\left(\left|\left(\frac{V}{\langle a, b\rangle}\right)^{*}\right|-1\right) \\
& =\left(q^{n-1}-q\right)\left(q^{n-2}-1\right),
\end{aligned}
$$

where $\left(\frac{V}{\langle a\rangle}\right)^{*}$ is the vector space of all linear functionals from $\frac{V}{\langle a\rangle}$ to $F$ and $\left(\frac{V}{\langle a, b\rangle}\right)^{*}$ is defined similarly. It follows that

$$
k=\frac{(q-1)\left(q^{n-1}-1\right)+q\left(q^{n-2}-1\right)^{2}}{q-1}-1 .
$$

Theorem 2.5. (1) For $\operatorname{dim}(V)=2, \Gamma$ is disconnected.

(2) For $\operatorname{dim}(V)=3, \operatorname{diam}(\Gamma)=3$.

(3) For $\operatorname{dim}(V)>3$, $\operatorname{diam}(\Gamma)=2$.

Proof. Let $T_{a, f}$ and $T_{b, g}$ be two transvections on $V$ with fixed hyperplanes $W_{1}=\operatorname{ker} f$ and $W_{2}=\operatorname{ker} g$, respectively. If $\operatorname{dim}(V)=2$ then $\operatorname{dim}(\operatorname{ker} f)=1$. Since $a \in \operatorname{ker} f, b \in \operatorname{ker} f$ if and only if $\langle b\rangle=\langle a\rangle$. It follows that $\Gamma$ is disconnected.

Suppose that $\operatorname{dim}(V)=3$. If $W_{1}=W_{2}$ then $d\left(T_{a, f}, T_{b, g}\right)=1$. If $W_{1} \neq W_{2}$ then $W_{1} \cap W_{2} \neq$ 0 and there exists a nonzero element $u \in W_{1} \cap W_{2}$. Since $\operatorname{dim}(\langle u, b\rangle) \leq 2$, there is $\gamma: V \longrightarrow F$ such that $\langle u, b\rangle \subseteq \operatorname{ker} \gamma$. It follows that $\left[T_{b, g}, T_{u, \gamma}\right]=1$. Also there exists $\delta: V \longrightarrow F$ such that $\langle u, a\rangle \subseteq \operatorname{ker} \delta$. Hence $\left[T_{a, f}, T_{u, \delta}\right]=1$. Also $\left[T_{u, \gamma}, T_{u, \delta}\right]=1$, thus $d\left(T_{a, f}, T_{b, g}\right) \leq 3$ and $\operatorname{diam}(\Gamma) \leq 3$. Now we show that $\operatorname{diam}(\Gamma)=3$. There exist 
transvections $T_{v_{1}, f_{1}}$ and $T_{v_{2}, f_{2}}$, where $\operatorname{ker} f_{1}=W_{1}$ and ker $f_{2}=W_{2}$, such that $v_{1} \notin W_{2}$ and $v_{2} \notin W_{1}$. If there exists a transvection $T_{u, h}$, where $\operatorname{ker} h=W$, such that $\left[T_{v_{1}, f_{1}}, T_{u, h}\right]=1$ and $\left[T_{v_{2}, f_{2}}, T_{u, h}\right]=1$ then $v_{1}, v_{2} \in W$. Since $\left\langle v_{1}\right\rangle \neq\left\langle v_{2}\right\rangle$ and $\operatorname{dim}(W)=2$, we obtain $W=\left\langle v_{1}, v_{2}\right\rangle$. It follows that $u=\lambda_{1} v_{1}+\lambda_{2} v_{2}$ and hence $u-\lambda_{1} v_{1} \in W_{2}$. This is a contradiction. Hence $\operatorname{diam}(\Gamma)=3$.

Now assume that $\operatorname{dim}(V)>3$. If $W_{1}=W_{2}$ then $d\left(T_{a, f}, T_{b, g}\right)=1$. If $W_{1} \neq W_{2}$ then $W_{1} \cap W_{2} \neq 0$ and there exists a nonzero element $u \in W_{1} \cap W_{2}$. Since $\operatorname{dim}(\langle u, a, b\rangle) \leq 3$, there is $\mu: V \longrightarrow F$ such that $\langle u, a, b\rangle \subseteq \operatorname{ker} \mu$. Now $\left[T_{a, f}, T_{u, \mu}\right]=1$ and $\left[T_{b, g}, T_{u, \mu}\right]=1$. This implies that $d\left(T_{a, f}, T_{b, g}\right) \leq 2$. Hence $\operatorname{diam}(\Gamma) \leq 2$. Now we show that $\operatorname{diam}(\Gamma)=2$. Let $W_{1}, W_{2}$ be two distinct hyperplanes, $v_{1} \in W_{1}-W_{2}$ and $v_{2} \in W_{2}-W_{1}$. Notice that $\left[T_{v_{1}, f_{1}}, T_{v_{2}, f_{2}}\right] \neq 1$, where $\operatorname{ker} f_{1}=W_{1}$ and ker $f_{2}=W_{2}$. Thus $\operatorname{diam}(\Gamma)=2$.

Theorem 2.6. $\omega(\Gamma)=\frac{\left(q^{k}-1\right)\left(q^{n-k}-1\right)}{q-1}$ where $k=\left[\frac{n}{2}\right]$.

Proof. We have $\left[T_{a_{i}, f_{i}}, T_{a_{j}, f_{j}}\right]=1$ for all $1 \leq i, j \leq t$ if and only if $a_{1}, a_{2}, \ldots, a_{t} \in$ $\bigcap_{i=1}^{t}$ ker $f_{i}$. So $\left\{T_{a_{1}, f_{1}}, T_{a_{2}, f_{2}}, \ldots, T_{a_{t}, f_{t}}\right\}$ is a complete subgraph of $\Gamma$ if and only if there exists a subspace $W$ of $V$ such that $a_{1}, a_{2}, \ldots, a_{t} \in W$ and $W \subseteq \operatorname{ker} f_{i}$ for all $1 \leq i \leq t$. It is sufficient to calculate $\left|S_{W}\right|$. Let $U_{1}, U_{2}, \ldots, U_{n-1}$ be subspaces of $V$ with $\operatorname{dim}\left(U_{i}\right)=i$. By Lemma 2.1, $\left|S_{U_{1}}\right|=\left|S_{U_{n-1}}\right|<\left|S_{U_{2}}\right|=\left|S_{U_{n-2}}\right|<\cdots$. Therefore $\omega(\Gamma)=\left|S_{U_{\left[\frac{n}{2}\right]}}\right|$.

Corollary 2.7. If $\operatorname{dim}(V) \geq 3$, then the girth of $\Gamma$ is equal to 3 .

Theorem 2.8. For $\operatorname{dim}(V)>2, \Gamma$ is not planar.

Proof. Let $W$ be a hyperplane of $V$. By Lemma 1.6, $\tau(W) \cong W$ and we have a complete subgraph $K_{|W|-1}$. Hence if $q^{n-1}-1 \geq 5$ then $\Gamma$ is not planar. If $q^{n-1}-1<5$ then $q=2, n=3$ and $V=\mathbb{Z}_{2} \times \mathbb{Z}_{2} \times \mathbb{Z}_{2}$. Let $W_{1}=\mathbb{Z}_{2} \times \mathbb{Z}_{2} \times 0, W_{2}=\mathbb{Z}_{2} \times 0 \times \mathbb{Z}_{2}$, $W_{3}=0 \times \mathbb{Z}_{2} \times \mathbb{Z}_{2}, W_{4}=\langle(1,0,0),(0,1,1)\rangle, W_{5}=\langle(1,0,1),(0,1,0)\rangle$ be hyperplanes of $V$. Set $A_{i}=\left\{T_{a, f} \mid a \in \operatorname{ker} f=W_{i}\right\}$. For all $u \in W_{i} \cap W_{j}$, there exist $T_{u, f_{i}} \in A_{i}, T_{u, f_{j}} \in A_{j}$. Then, with contraction of $A_{i}$, where $1 \leq i \leq 5$, we obtain a complete graph $K_{5}$. This completes the proof.

Theorem 2.9. $\Gamma$ is perfect if and only if $\operatorname{dim}(V)=3$.

Proof. Let $\operatorname{dim}(V)=3$. Suppose that $\Gamma$ has an induced cycle of length $m \geq 4$. Also assume that $T_{v_{1}, f_{1}}, T_{v_{2}, f_{2}}, T_{v_{3}, f_{3}}$ are three consecutive vertices of this cycle, where ker $f_{i}=W_{i}$ for all $1 \leq i \leq 3$. We have $v_{1} \in W_{1} \cap W_{2}, v_{2} \in W_{1} \cap W_{2} \cap W_{3}, v_{3} \in W_{2} \cap W_{3}$. If $W_{i} \neq W_{j}$ for all $1 \leq i, j \leq 3,1 \leq \operatorname{dim}\left(W_{1} \cap W_{2} \cap W_{3}\right) \leq \operatorname{dim}\left(W_{1} \cap W_{2}\right)=1$, then $W_{1} \cap W_{2} \cap W_{3}=W_{1} \cap W_{2}$. Similarly, $W_{1} \cap W_{2} \cap W_{3}=W_{2} \cap W_{3}$. Hence $W_{1} \cap W_{2}=W_{2} \cap W_{3}$. Thus $v_{1} \in W_{3}$ and $v_{3} \in W_{1}$, a contradiction. Hence $W_{1}=W_{2}$ or $W_{2}=W_{3}$. Since $m \geq 4, W_{1} \neq W_{3}$. Concequently we can assume $W_{1}=W_{2}, W_{3}=W_{4}, W_{5}=W_{6}, \cdots$. If $m$ is odd, then $T_{v_{m-1}, f_{m-1}}, T_{v_{m}, f_{m}}, T_{v_{1}, f_{1}}$ are three consecutive vertices and, $W_{1}=W_{2}, W_{m-1}=W_{m-2}$. Then $W_{m} \neq W_{1}$ and $W_{m} \neq W_{m-1}$, a contradiction. Thus $m$ is even and $\Gamma$ has no odd induced cycle of length at least five. It follows that $\Gamma$ is perfect.

Now let $\operatorname{dim}(V)=4$ and $V=\left\langle v_{1}, v_{2}, v_{3}, v_{4}\right\rangle$. Assume that $W_{1}=\left\langle v_{1}, v_{2}, v_{3}\right\rangle, W_{2}=$ $\left\langle v_{1}, v_{2}, v_{4}\right\rangle$ and $\bar{W}=\left\langle v_{1}, v_{3}, v_{4}\right\rangle$. Since $\left|V-\left(W_{1} \cup W_{2} \cup \bar{W}\right)\right|=q^{4}-3 q^{3}+3 q^{2}-q=$ $q(q-1)^{3}>0$, there is $v_{5} \in V-\left(W_{1} \cup W_{2} \cup \bar{W}\right)$. Set $W_{3}=\left\langle v_{2}, v_{4}, v_{5}\right\rangle, W_{4}=\left\langle v_{3}, v_{4}, v_{5}\right\rangle$ and $W_{5}=\left\langle v_{1}, v_{3}, v_{5}\right\rangle$. If $v_{4} \in W_{5}$ then $v_{4}=\lambda_{1} v_{1}+\lambda_{3} v_{3}+\lambda_{5} v_{5}$. Since $v_{1}, v_{3}, v_{4}$ are independent, we have $\lambda_{5} \neq 0$ and $v_{5} \in \bar{W}$, which is a contradiction. Thus $v_{4} \notin W_{5}$. Also $v_{4} \notin W_{1}$. Hence $v_{4} \notin W_{1} \cap W_{5}$. Similarly, $v_{3} \notin W_{3} \cap W_{2}, v_{1} \notin W_{3} \cap W_{4}, v_{2} \notin W_{4} \cap W_{5}$, $v_{5} \notin W_{1} \cap W_{2}$. Now $T_{v_{1}, f_{1}}, T_{v_{2}, f_{2}}, T_{v_{4}, f_{3}}, T_{v_{5}, f_{4}}, T_{v_{3}, f_{5}}$ forms an induced cycle of length 5 , where $\operatorname{ker} f_{i}=W_{i}$ for all $1 \leq i \leq 5$. Since the complement of any induced cycle of length 5 in $\Gamma$ is an induced cycle of length 5 in $\bar{\Gamma}, \Gamma$ and $\bar{\Gamma}$ have induced cycles of length 5 . Concequently $\Gamma$ is not perfect.

Now assume that $\operatorname{dim}(V) \geq 5$ and $V=\left\langle v_{1}, \ldots, v_{5}\right\rangle \oplus W$. Suppose that $W_{i}=$ 
$\left\langle\left\{v_{1}, v_{2}, v_{3}, v_{4}, v_{5}\right\}-\left\{v_{i}\right\}\right\rangle \oplus W$ for $1 \leq i \leq 5$. Then $T_{v_{1}, f_{5}}, T_{v_{4}, f_{3}}, T_{v_{2}, f_{1}}, T_{v_{5}, f_{4}}, T_{v_{3}, f_{2}}$ forms an induced cycle of length 5 , where $\operatorname{ker} f_{i}=W_{i}$ for all $1 \leq i \leq 5$. Concequently, both $\Gamma$ and $\bar{\Gamma}$ have induced cycles of length 5 and $\Gamma$ is not perfect.

Corollary 2.10. If $\operatorname{dim}(V)=3$, then $\chi(\Gamma)=\omega(\Gamma)=q^{2}-1$.

Theorem 2.11. For $n \geq 3, \gamma(\Gamma) \leq \min \left\{(q+1)^{2}, \frac{q^{n}-1}{q-1}\right\}$ and for $q=2$ or $n \geq 5$, $\gamma(\Gamma) \geq q^{2}$.

Proof. For each hyperplane $W$, let $a_{W}$ be a nonzero element of $W$ and $f_{W}$ be a linear functional with $\operatorname{ker} f_{W}=W$. Set $S=\left\{T_{a_{W}, f_{W}} \mid \mathrm{W}\right.$ is a hyperplane of $\left.V\right\}$. Let $T_{b, g} \in V(\Gamma)$ and $\operatorname{ker} g=W_{1}$. Then $T_{a_{W_{1}}, f_{W_{1}}} \in S$ and $\left[T_{b, g}, T_{a_{W_{1}}, f_{W_{1}}}\right]=1$. Hence $S$ is a dominating set for $\Gamma$, and so $\gamma(\Gamma) \leq \frac{q^{n}-1}{q-1}$. Now assume that $\operatorname{dim}(V) \geq 4$. Let $W$ be a subspace of $V$ with $\operatorname{dim}(W)=n-2$. Observe that $V$ has $q+1$ hyperplanes $W_{1}, W_{2}, \ldots, W_{q+1}$ containing $W$. Let $f_{1}, f_{2}, \ldots, f_{q+1}$ be linear functionals with $\operatorname{ker} f_{j}=W_{j}$ for $j=1,2, \ldots, q+1$. Clearly, we have $V=W_{1} \cup W_{2} \cup \ldots \cup W_{q+1}$. Let $U$ be a subspace of $W$ with $\operatorname{dim}(U)=2$ and let $\left\langle a_{1}\right\rangle,\left\langle a_{2}\right\rangle, \ldots,\left\langle a_{q+1}\right\rangle$ be all distinct one dimensional subspaces of $U$. We claim now that $S=\left\{T_{a_{i}, f_{j}} \mid i, j \in\{1,2, \ldots, q+1\}\right\}$ is a dominating set for $\Gamma$. For an ordinary transvection $T_{b, g}$, there exists $W_{j}$ such that $b \in W_{j}$. Since $\operatorname{dim}(U \cap \operatorname{ker} g)=1$, there exists $a_{i} \in \operatorname{ker} g$. It then follows that $\left[T_{a_{i}, f_{j}}, T_{b, g}\right]=1$, which proves the claim. Thus, we get $\gamma(\Gamma) \leq|S|=(q+1)^{2}$ as desired. Now suppose $\gamma(\Gamma)=t$. Since $\Gamma$ is $k$-regular, we have

$$
\begin{aligned}
t & \geq \frac{|V(\Gamma)|}{k}=\frac{\left(q^{n}-1\right)\left(q^{n-1}-1\right)}{(q-1)\left(q^{n-1}-1\right)+\left(q^{n-1}-q\right)\left(q^{n-2}-1\right)-(q-1)} \\
& \geq \frac{q^{2 n-1}-q^{n}-q^{n-1}+1}{q^{2 n-3}+q^{n}-3 q^{n-1}-q+2} \\
& >\frac{q^{2 n-1}-q^{n}-q^{n-1}}{q^{2 n-3}+q^{n}-3 q^{n-1}} \\
& >\frac{\left(q^{n}-q-1\right)}{\left(q^{n-2}+q-3\right)} .
\end{aligned}
$$

But for $q=2$ or $n \geq 5, \frac{\left(q^{n}-q-1\right)}{\left(q^{n-2}+q-3\right)}>q^{2}-1$. Hence $q^{2} \leq \gamma(\Gamma)$. This completes the proof.

Theorem 2.12. If $q$ is odd, then $\Gamma$ has a 1 -factor and $\alpha(\Gamma) \leq \frac{\left(q^{n}-1\right)\left(q^{n-1}-1\right)}{(q-1)\left(q^{\left[\frac{n}{2}\right]}-1\right)\left(q^{n-\left[\frac{n}{2}\right]}-1\right)}$.

Proof. It follows from Theorem 1.8.

Example 2.13. Let $G=G L(3,2)$. Then the commuting graph $\Gamma=\mathcal{C}(G, X)$ satisfies the following conditions:
(1) $|V(\Gamma)|=21$.
(2) $\Gamma$ is 4-regular.
(3) $|E(\Gamma)|=42$.
(4) $\operatorname{diam}(\Gamma)=3$.
(5) $\Gamma$ is perfect.
(6) $\chi(\Gamma)=\omega(\Gamma)=3$.
(7) $\gamma(\Gamma)=5$.

Proof. Parts (1) - (6) are clear. For part (7), by Theorem 2.11, we have $4 \leq \gamma(\Gamma) \leq 7$. Since

$4 \times 5 \leq|V(\Gamma)|=21, \gamma(\Gamma) \geq 5$. Observe that $\left\{T_{v_{1}, f_{1}}, T_{v_{2}, f_{2}}, T_{v_{3}, f_{3}}, T_{v_{5}, f_{5}}, T_{v_{6}, f_{6}}\right\}$ is a dominating set, where $v_{1}=(1,0,0), v_{2}=(1,0,1), v_{3}=(0,1,1), v_{5}=(1,1,1), v_{6}=$ $(1,1,0)$ and ker $f_{1}=\mathbb{Z}_{2} \times \mathbb{Z}_{2} \times 0$, ker $f_{2}=\mathbb{Z}_{2} \times 0 \times \mathbb{Z}_{2}$, ker $f_{3}=0 \times \mathbb{Z}_{2} \times \mathbb{Z}_{2}$, ker $f_{5}=$ $\langle(1,0,1),(0,1,0)\rangle$, ker $f_{6}=\langle(1,1,0),(0,0,1)\rangle$. Thus $\gamma(\Gamma)=5$. 
Acknowledgment. The authors are deeply grateful to the referee for his/her valuable comments and suggestions, which helped us to improve the paper.

\section{References}

[1] P.J. Cameron and Q. Mary, Automorphisms of graphs, London E14Ns, U.K. Draft, 2001.

[2] M. Chudnovsky, N. Robertson, P. Seymour and R. Thomas, The strong perfect graph theorem, Ann. of Math. (2), 164 (1), 51-229, 2006.

[3] H. Poliatsek, Irreducible Groups Generated by Transvections over Finite Fields of Characteristic Two, J. Algebra, 39, 328-333, 1976.

[4] J.J. Rotman, An Introduction to the Theory of groups, Springer-Verlag Newyork, Inc. 1995. 